\title{
Respuesta temporal al ambiente lumínico y la sequía inducida en el regenerado de una masa mixta en el entorno mediterráneo
}

Temporary response to the light environnment and induced drought on the regeneration of a mixed forest in the Mediterranean environment

\author{
Marta Pardos ${ }^{1}$, Guillermo Madrigal ${ }^{2}$, Javier de Dios-García ${ }^{3}$, \\ Javier Gordo ${ }^{4}$, Rafael Calama ${ }^{5}$ \\ ${ }^{1,2,3,4}$ Departamento de Dinámica y Gestión Forestal, INIA-CIFOR, iuFOR, \\ crtraA Coruña Km 7,5, 28040-Madrid (pardos@inia.es) \\ ${ }^{2}$ Servicio Territorial de Medio Ambiente de Valladolid. \\ C/ Duque de la Victoria 8, 47001-Valladolid (goralofr@jcyl.es)
}




\title{
Resumen
}

El trabajo se desarrolla en las masas mixtas de pino piñonero, sabina y encina de los páramos calizos de la Meseta Norte. Se estudia la supervivencia, crecimiento, respuesta fisiológica y reparto de biomasa de una plantación de las tres especies a lo largo de un gradiente lumínico y bajo condiciones de sequía inducida. Se establecieron 12 parcelas $\left(45 \mathrm{~m}^{2}\right)$, divididas en dos subparcelas, a su vez con 4 bloques, donde se plantaron 9 plantas ( 3 plantas por especie). Se fijaron 3 niveles de luz (HL: GSF 0.75: ML: GSF 0.45; LL: GSF 0.26); y dos niveles de disponibilidad hídrica (Co: condiciones ambientales naturales; y W-: reducción de precipitación del 30\% mediante sistema de exclusión de precipitación). El ensayo duró desde diciembre 2011 hasta mayo 2015. Se evaluó la supervivencia en 19 ocasiones y se midió la fluorescencia a la clorofila 4 veces. En mayo 2015 se hizo cosecha final, analizándose el reparto de biomasa y crecimiento. Las mayores tasas de mortalidad se registraron tras el primer verano, con efecto negativo del tratamiento HL sobre la supervivencia de encina y pino, y poco efecto de la exclusión. Bajo sombra, las tasas de supervivencia fueron mayores. La supervivencia estuvo íntimamente relacionada con su comportamiento fisiológico, modificado por el ambiente lumínico y la época del año, pero no por la exclusión. Los resultados apuntan a la mejor capacidad adaptativa de la sabina y a un peor comportamiento de la encina. Sin embargo, la capacidad rebrotadora de la encina puede resultarle beneficiosa a medio-largo plazo.

Palabras clave: ensayo de exclusión, fluorescencia de la clorofila, Juniperus thurifera, Pinus pinea, Quercus ilex.

\begin{abstract}
The study was set up in the mixed stands in the limestone plain areas of the Northern Plateaus of Spain. The aim was to assess the effect of a natural light gradient and a throughfall exclusion experiment on survival, growth, physiology and biomass allocation in a plantation done with one-year old plants of stone pine, holm oak and juniper. Plants were distributed in twelve $45 \mathrm{~m}^{2}$ fenced plots, divided in two subplots with four blocks, where nine plants were planted (3 plants per species). Three light levels (HL: GSF 0.75: ML: GSF 0.45; LL: GSF 0.26) and two levels of water availability (Co: current climate conditions and W-: $30 \%$ rainfall) were selected. The study lasted from December 2011 to May 2015. We assessed survival in 19 occasions and chlorophyll fluorescence was measured in four different dates. Seedlings were harvested in May 2015 and growth and biomass allocation were analyzed. The highest mortality was recorded during the first summer. There was a negative effect of high light on survival of holm oak and stone pine, while the effect of rainfall exclusion was small. Under shade, survival rates were higher. Survival was intimately related to physiology that was modified by light environment and date of measurement, but not by rainfall exclusion. Our results highlight the better adaptive ability of juniper and the lower performance of holm oak. However, the resprouting ability of holm oak will benefit the species in the mid-long term.
\end{abstract}

Keywords: throughfall exclusion experiment, chlorophyll fluorescence Juniperus thurifera, Pinus pinea, Quercus ilex. 


\section{Introducción}

En los ambientes mediterráneos continentales la luz y el agua son los dos principales factores ecológicos que controlan la distribución de especies y la dinámica de la regeneración natural, de tal manera que su interacción puede modificar la productividad y composición de sus masas (Quero et al., 2006). En general, estas masas se caracterizan por la presencia de sólo dos a cuatro especies arbóreas, en las que el regenerado presenta características morfológicas y fisiológicas divergentes, así como una considerable variabilidad funcional dentro de cada especie en respuesta a condiciones variables de luz y disponibilidad hídrica (Valladares et al., 2015). En estos ambientes, la vulnerabilidad del regenerado a la cavitación por sequía está íntimamente relacionada con las características estructurales de las hojas y el tallo (Granda et al., 2014; Zhang et al., 2017). Por ello, cabe esperar que el regenerado presente un comportamiento diferente, en términos de crecimiento y supervivencia, de acuerdo con sus estrategias reproductivas y sus mecanismos de adaptación a estreses ambientales (Mayoral et al., 2016). Sin embargo, estos ecosistemas con una diversidad de especies tan reducida son especialmente vulnerables al cambio global, debido al reducido número de especies que contribuyen al funcionamiento y productividad de los mismos. Por tanto, la pérdida de una especie, que no podrá ser sustituida por otra, puede tener consecuencias muy negativas en su ecología funcional (Valladares et al., 2015).

Los ecosistemas mediterráneos presentan una elevada resiliencia a episodios de sequía extrema (Saunier, 2018). Sin embargo, todas las proyecciones climáticas para la región mediterránea coinciden en las predicciones sobre sequías estivales más prolongadas, incluyendo episodios extremos (olas de calor y sequías extremas) más frecuentes (Sperlich et al., 2015; Pardos y Calama, 2017). Dichas proyecciones también predicen la reducción en las precipitaciones de primavera y verano entre el 20 y el 40\%, según los escenarios de cambio climático (Besson et al., 2014). Las limitaciones crónicas de recursos, como es la reducción de la precipitación, tiene efectos progresivos y acumulativos a lo largo del tiempo, especialmente en ecosistemas forestales con especies arbóreas (Smith et al., 2009). A la larga, las sequías más intensas están forzando a las especies arbóreas a desplegar su máxima plasticidad fenotípica. Los mecanismos de resistencia a la sequía, de respuesta a sequías extremas y severas y su subsiguiente recuperación incluyen procesos desde el nivel molecular al de masa, que operan a diferentes escalas temporales (Barbeta y Peñuelas, 2015). A nivel fisiológico, el cierre estomático es el proceso más rápido que limita la transpiración y evita daños irreversibles en el sistema hidráulico, que conducen a la muerte de la planta (Limousin et al., 2010). Por tanto, nuestra capacidad para anticipar el futuro de estos ecosistemas se basa en conocer la respuesta a la sequía para un rango amplio de condiciones ambientales (MartinStPaul et al., 2013); y esto resulta de especial interés en la región mediterránea, donde la respuesta in situ de las especies a periodos de sequía recurrentes y prolongados no es del todo conocida.

Las masas heterogéneas de Pinus pinea-Quercus-Juniperus de los páramos ca- 
lizos de la Meseta Norte en las que se centra este estudio se sitúan en una región de escasos recursos hídricos, sometida a una sequía estival prolongada, a lo que se unen las elevadas temperaturas y la alta insolación estival (Pardos et al., 2010; de Dios García et al., 2018). Estas condiciones agravan el hecho de que estas masas heterogéneas presenten problemas de regeneración (Manso et al., 2014), de tal forma que las proyecciones sobre sequías estivales más frecuentes, prolongadas y severas en la región afectarán negativamente al establecimiento de las plantas a corto plazo y a la regeneración de la masa a largo plazo.

Las tres especies objeto de estudio (Pinus pinea, Quercus ilex y Juniperus thurifera) son especialmente sensibles, durante el estado juvenil, a las sequías prolongadas cuando crecen en espacios abiertos (Pardos et al., 2014). De hecho, la supervivencia del regenerado se ve muy negativamente afectada por la sequía estival en Quercus ilex (Ogaya y Peñuelas, 2007) y Pinus pinea (Mayoral et al., 2015). Estas especies ocupan diferentes nichos ecológicos dentro de la masa mixta, debido a sus diferentes estrategias para hacer frente a la sequía y a su diferente preferencia por la luz (Mayoral et al., 2016). Su capacidad para ajustar su fisiología en términos de supervivencia, crecimiento y caracteres funcionales indicará su diferente nivel de aclimatación y permitirá adquirir un mejor conocimiento sobre los caracteres que contribuyen a una mayor resistencia a niveles diferentes de luz y disponibilidad hídrica para predecir la distribución de las especies ante fluctuaciones ambientales (Kerr et al., 2015). El análisis de esos caracteres y su variación entre especies resulta especialmente relevante para la coexistencia futura de las especies y sus dinámicas poblacionales.

Este estudio tiene como objetivo estudiar la respuesta del regenerado de las tres especies en términos de supervivencia, crecimiento, respuesta fisiológica y reparto de biomasa a lo largo de tres años, en una plantación realizada en un gradiente lumínico natural, considerando un ensayo de exclusión de la precipitación, que reduce la misma en un $30 \%$, lo que se corresponde con la reducción predicha en la precipitación estival para el entorno mediterráneo (escenario RCP 8.5, IPCC 2013). Los ensayos de exclusión de precipitación han sido identificados como una forma de determinar cómo los cambios en la precipitación afectan al funcionamiento de los ecosistemas (Martin-StPaul et al., 2013). Sin embargo, como estos ensayos están limitados en el tiempo y en el espacio hay que tener precaución a la de hora de sacar conclusiones de los mismos para escalas temporales y espaciales mayores (Rustad, 2008; Leuzinger et al., 2011). Nuestra hipótesis es que la respuesta del regenerado de las especies estudiadas, en términos de supervivencia, a diferentes condiciones ambientales de luz y de disponibilidad hídrica será específica de cada especie y vendrá muy condicionada por las condiciones climáticas. Además, las diferentes estrategias de uso del agua y la luz de las especies permitirán la coexistencia del regenerado establecido, gracias a la complementariedad y facilitación bajo condiciones ambientales variables. En este sentido, esperamos encontrar:

1. Una respuesta morfológica plástica en Quercus ilex, debido a su comportamiento anisohídrico y su capacidad rebrotadora. 
2. Una estrategia conservadora en Pinus pinea.

3. Una elevada plasticidad fisiológica en Juniperus thurifera, lo que facilitará su supervivencia.

\section{Material y métodos}

Este estudio se lleva a cabo en las mixtas mixtas de Pinus pinea-Quercus-Juniperus de los páramos calizos de la Meseta Norte, donde ocupan una superficie de $11000 \mathrm{ha}$, en un rango de altitud de 800-850 m. En concreto, el estudio se lleva a

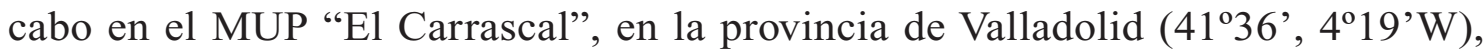
donde Pinus pinea (pino piñonero) convive con Juniperus thurifera (sabina albar) y Quercus ilex (encina). Hasta 1980 estas masas estuvieron en manos privadas, por lo que la falta de una gestión enfocada a favorecer al Pinus pinea para la producción de piña ha dado lugar a unas masas heterogéneas, que actualmente se gestionan para el mantenimiento de dicha diversidad.

El clima es mediterráneo continental, con temperatura media anual de $11^{\circ} \mathrm{C}$, registrándose una marcada sequía estival, que se alarga durante cuatro meses, con temperaturas máximas que pueden alcanzar $\operatorname{los} 45^{\circ} \mathrm{C}$, y temperaturas relativamente frías en invierno (mínimas absolutas de $-15^{\circ} \mathrm{C}$ ). Existe, además, una gran variabilidad interanual en las precipitaciones, que pueden variar entre 220 y $630 \mathrm{~mm}$ anuales y precipitaciones estivales de $21 \mathrm{~mm}$ (Calama y Montero 2007). Los suelos son inceptisoles, con una capacidad de retención de agua de $176 \mathrm{~mm}$.

En diciembre 2011 se instalaron 12 parcelas valladas de $45 \mathrm{~m}^{2}\left(5 \times 9 \mathrm{~m}^{2}\right)$ que cubrían el rango de condiciones de luz y suelo de la zona, siguiendo un diseño factorial. Cada parcela se dividió en dos subparcelas de $4 \mathrm{~m} \times 4 \mathrm{~m}$, divididas a su vez en 4 bloques de $2 \mathrm{~m} \times 2 \mathrm{~m}$, donde se plantaron 9 plantas ( 3 plantas por especie). Se fijaron 3 niveles de disponibilidad de luz, basándose en los valores de GSF (alto HL, con GSF de 0.75 ; medio ML, con GSF de 0.45 ; y bajo LL, con GSF de 0.26), identificándose 4 parcelas por nivel de luz. GSF (Global Site Factor) se calcula basándose en una transmisividad de la luz de 0.8 y una relación de radiación directa: radiación difusa de $0.1 \mathrm{GSF}$, empleando el software Hemiview 2.1 para su análisis (Delta-T Devices, Ltd, Cambridge, UK). Se tomaron dos fotografías hemisféricas por parcela para caracterizar el entorno lumínico de las mismas.

Se distinguieron, además, dos niveles de disponibilidad hídrica, uno (control, Co) correspondiente a las condiciones ambientales naturales y otro (sequía inducida, W-) en el que se estableció una reducción de precipitación del 30\%, que corresponde con la reducción predicha en la precipitación estival para el entorno mediterráneo (escenario RCP 8.5, IPCC 2013). Con tal fin, se instalaron un sistema de exclusión de precipitación consistente en unos marcos metálicos de $1.5 \mathrm{~m}$ x $3 \mathrm{~m}$ encima de los cuales se colocaron una piezas de metacrilato en forma de $\mathrm{V}$, sin filtro de rayos UV (Barlocast ${ }^{\circledR}$, Faberplast SL, Madrid) (ver Figura 1 con la representación esquemática del ensayo). Los sistemas de exclusión se instalaron en cada sub- 
parcela de 6 de las 12 parcelas, por tanto cubriendo 2 subparcelas por tratamiento de luz. En diciembre de 2011 se plantaron 864 plantas de una savia (12 parcelas x 2 subparcelas $\mathrm{x} 4$ bloques $\mathrm{x} 3$ especies $\mathrm{x} 3$ plantas), suministradas por el vivero Central de la Junta de Castilla y León. El sistema de exclusión se retrasó a marzo de 2013 debido a las condiciones de sequía marcadas del 2012, que obligaron a regar a mediados de julio y septiembre con $12.71 \mathrm{~m}^{-2}$ en cada ocasión. A pesar del riego, la mortalidad a finales de 2012 fue del 47\%. El ensayo se prolongó hasta mayo 2015.

Los datos climáticos diarios se registraron en una estación meteorológica cercana. Además, se realizó un registro continuo de la humedad del suelo (primeros 20 $\mathrm{cm}$ ) en las subparcelas control y las de sequía inducida, de parcelas con alta y baja disponibilidad de luz mediante microestaciones HOBO (Micro HWS, ONSET, Massachussetts, USA); así como un registro continuo de la temperatura ambiental y de la intensidad de la luz a $20 \mathrm{~cm}$ sobre el suelo con un HOBO Pendant ${ }^{\circledR}$.

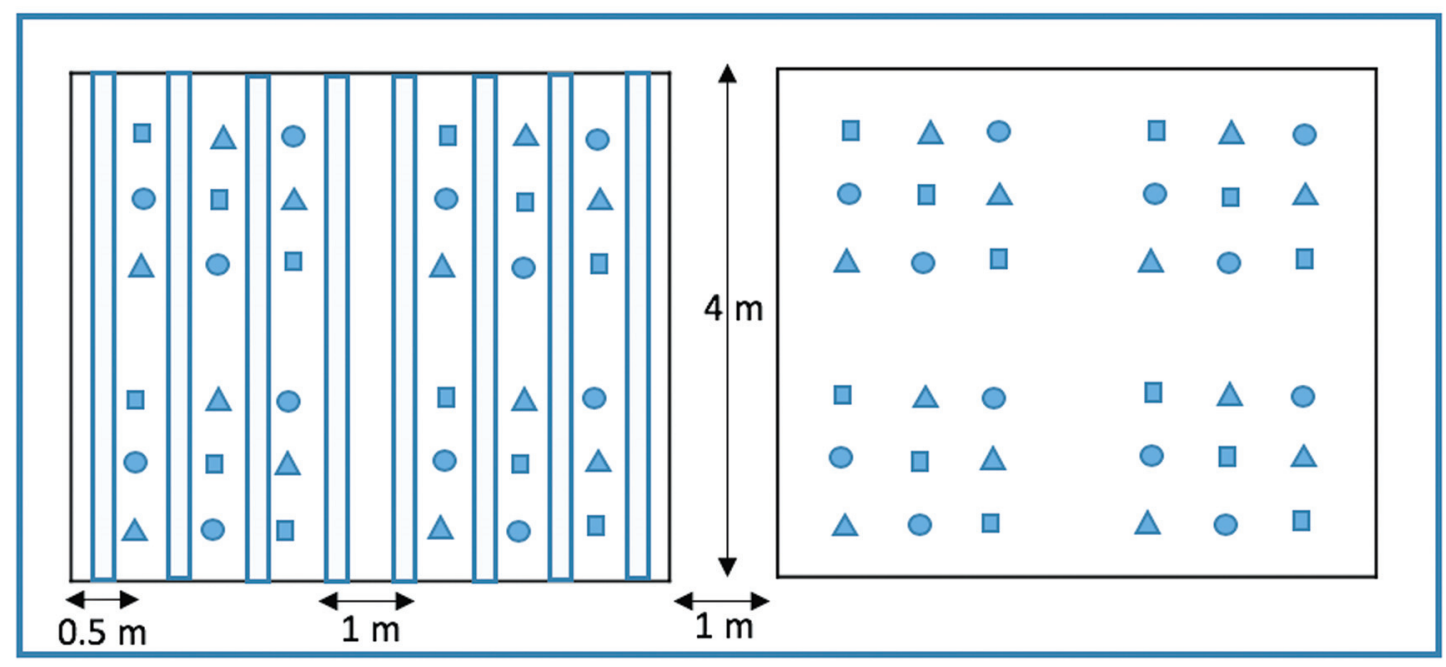

Figura 1. Representación esquemática de la plantación en una de las 6 parcelas instaladas con exclusión de precipitación. Cada parcela contiene dos subparcelas, con (izquierda) y sin (derecha) exclusión. Las tiras verticales grises a la izquierda representan los dispositivos de exclusión. Las especies se representan por cuadrados (pino), triángulos (encina) y círculos (sabina).

Se evaluó la supervivencia en 19 ocasiones, con una periodicidad de 50 días. Además, se realizaron medidas de fluorescencia a la clorofila en 4 ocasiones (J12013, Fb2014, J12014, My2015), en 4 plantas por especie, tratamiento y parcela (48 plantas en total), en las 6 parcelas donde se instalaron los sistemas de exclusión. La fluorescencia de la clorofila se midió a media mañana (10 am-12 pm) en el tallo principal de las plantas con un fluorímetro portátil de pulso modulado (FMS2, Hansatech Instruments Ltd, UK) El rendimiento fotoquímico del PSII (ФPSII) se calculó como $\Phi P S I I=\left(F^{\prime} m-F s\right) / F^{\prime} m$, donde $F^{\prime} m$ es la fluoresencia máxima con luz y Fs es el régimen estacionario de la fluorescencia adaptada a luz 
(Kramer et al., 2004). La eficiencia fotoquímica máxima del PSII (Fv/Fm) se calculó después de un periodo de adaptación a la oscuridad de 30 minutos. Al finalizar el ensayo en mayo 2015, se cosecharon las plantas vivas. Se excavaron y se conservaron en hielo para su transporte al laboratorio y posterior procesado. Se midieron las alturas, el diámetro y la superficie foliar, se separaron las fracciones de parte aérea y radical y se secaron en estufa a $70^{\circ} \mathrm{C}$, analizándose el reparto de biomasa (pesos secos de hojas, parte aérea, raíz y total) y su crecimiento. Se calcularon las relaciones de pesos de las distintas fracciones: peso seco parte aérea a raíz $(\mathrm{S} / \mathrm{R})$, peso seco hojas a parte aérea ( $\mathrm{PS}_{\text {hoj }} / \mathrm{PS}_{\text {aer }}$ ), peso seco parte aérea a total ( $\mathrm{PS}_{\text {aer }} / \mathrm{PS}_{\text {tot }}$ ), peso seco raíz a total ( $\mathrm{PS}_{\text {raiz }} / \mathrm{PS}_{\text {tot }}$ ), peso seco raíz principal a total ( $\mathrm{PS}_{\mathrm{rzpp}} / \mathrm{PS}$ tot), peso seco raíces secundarias a total (PS $\mathrm{Prz}^{2} / \mathrm{PS}_{\mathrm{tot}}$ ), así como SLA (relación superficie foliar a peso foliar).

El análisis del efecto de la especie, luz, exclusión y sus interacciones sobre la supervivencia se realizó mediante un modelo mixto lineal generalizado, asumiendo una distribución binomial $(0,1)$ para la variable de respuesta, e incluyendo un efecto aleatorio de parcela con el objetivo de considerar el anidamiento del efecto exclusión en el efecto luz. El análisis se realizó tanto para la supervivencia observada tras el primer verano (cuando aún no se había instalado el experimento de exclusión) como para el experimento en su totalidad (ya considerando el efecto de la exclusión).

Los análisis de los parámetros de fluorescencia de la clorofila y parámetros biométricos se realizaron mediante un modelo mixto lineal, incluyendo un efecto de parcela al objeto de considerar el anidamiento propio del diseño del experimento, empleándose el test de comparación de medias de Tukey $(\mathrm{P}$-valor $<0.05)$ para identificar diferencias significativas entre tratamientos.

Los análisis estadísticos se realizaron con SAS 9.2 (SAS Institute, Cary, NC, USA).

\section{Resultados}

Los datos obtenidos de las estaciones meteorológicas instaladas en las parcelas muestran una reducción de la humedad del suelo del $14.8 \%$ en el tratamiento de exclusión de la precipitación (W-) comparado con el control (Co), siendo el efecto de la exclusión menos significativo en las épocas más secas (verano 2013: P-valor= 0.0270 y otoño 2014: P-valor $=0.0937$ ). Además, aunque se observó en general una menor humedad del suelo en las parcelas con una mayor cobertura (LL), el efecto de la exclusión sobre la humedad del suelo fue más patente en las parcelas localizadas a plena luz (HL).

Los resultados de la supervivencia a lo largo del tiempo mostraron un efecto muy significativo de la fecha, de tal manera que las mayores tasas de mortalidad se produjeron en el primer verano tras la plantación (Figura 2), llegándose a alcanzar unas tasas de mortalidad entre el 7\% y 70\%, según la especie y el tratamiento. Para ese primer periodo se observó un efecto muy significativo tanto de la luz (P-valor 


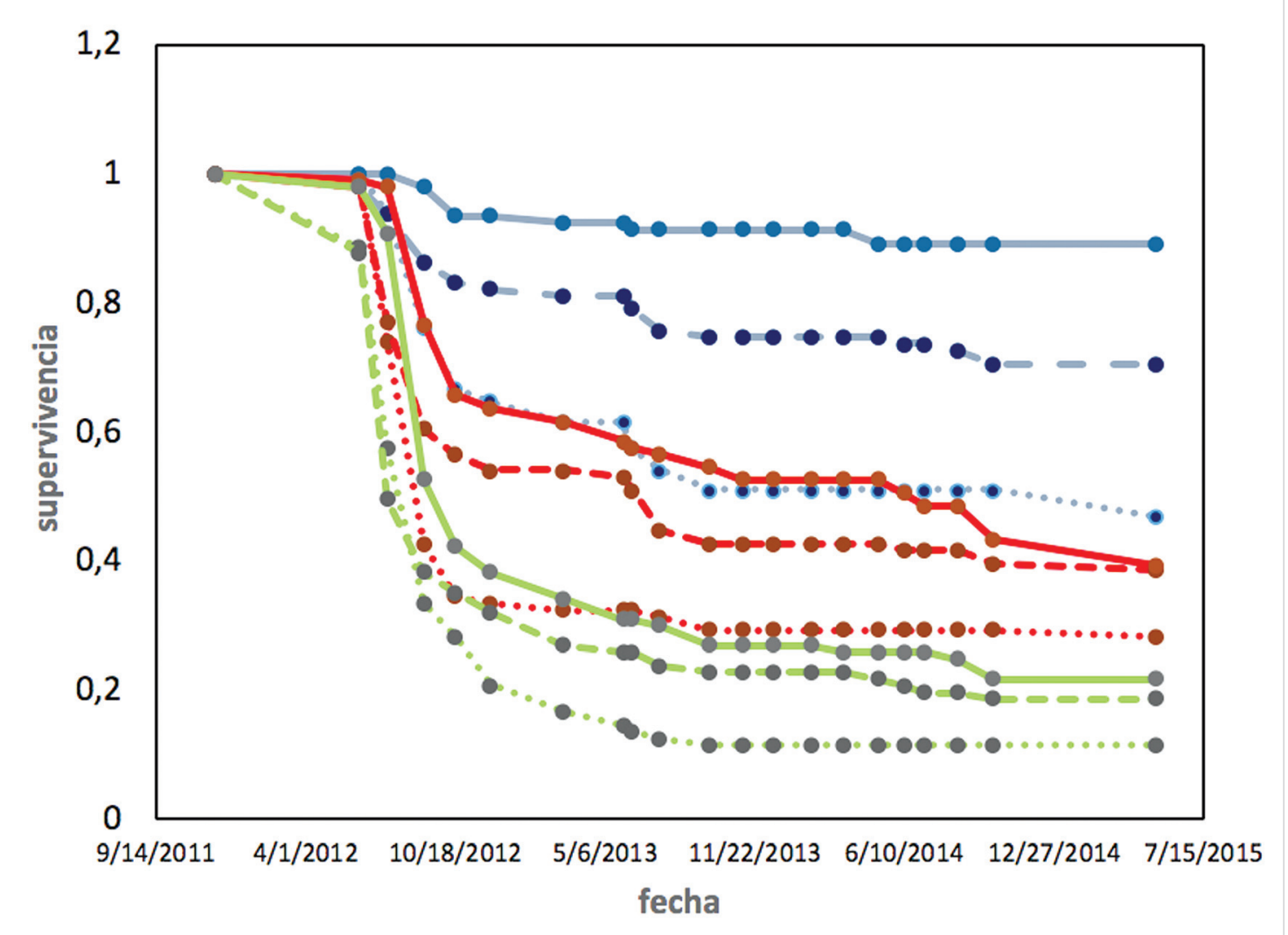

Figura 2. Evolución de la supervivencia desde diciembre 2011 hasta mayo de 2015 de una plantación de sabina (en azul), pino piñonero (en rojo) y encina (en verde), según el ambiente lumínico (— tratamiento LL; - - . tratamiento ML; ........ tratamiento HL).

$<0.0001)$ como de la especie (P-valor $<0.0001)$, no observándose efecto de la interacción $(\mathrm{P}$-valor $=0.5343)$. En el caso del ambiente lumínico se observaron valores menores de supervivencia a medida que disminuía la cobertura arbórea (43.1\% en HL, $58.2 \%$ en ML y $67.1 \%$ en LL), mientras que a nivel de especie, la supervivencia de la encina (35.2\%) se vio muy negativamente afectada tras el primer verano, seguido del pino (52.2\%) y por último de la sabina albar (80.9\%).

Tras este primer verano, las plantas de sabina albar bajo sombra (LL) mostraron unas tasas de supervivencia del $93.5 \%$, que contrastan con las tasas de supervivencia del $28.1 \%$ en las plantas de encina crecidas a pleno sol (HL). Si se realiza un análisis sobre el ensayo en su totalidad se observa un efecto no significativo de la exclusión $(\mathrm{P}$-valor $=0.7363)$ o de sus interacciones con la especie $(\mathrm{P}$-valor $=$ 0.3277) o la luz ( $\mathrm{p}$-valor $=0.6748)$, por lo que la exclusión se excluye del análisis. La supervivencia al final del ensayo se vio afectada por la especie (P-valor $<$ $0.0001)$ ( $17.2 \%$ en encina, $35.4 \%$ en pino piñonero y $68.6 \%$ en sabina albar) y el ambiente lumínico (P-valor $<0.0001)(28.8 .1 \%$ en $\mathrm{HL}, 42.5 \%$ en ML y $50.0 \%$ en LL). También debe indicarse un efecto significativo de la interacción luz x especie $(\mathrm{P}$-valor $=0.0112)$, que indica un comportamiento diferencial del pino piñonero $\mathrm{y}$ 
la encina, con una tendencia a equilibrar la supervivencia en los tratamiento LL y ML al final del periodo de estudio (Figura 1).

Con respecto al comportamiento fisiológico de las plantas en términos de fluorescencia a la clorofila se observó un efecto significativo de la fecha de medición (P-valor $<0.0001$ ), con valores significativamente mayores de ФPSII y Fv/Fm en verano (julio 2013) y mediados de primavera (mayo 2015), con respecto a los observados a principios de otoño (septiembre 2013) y mediados de invierno (febrero 2014) (Tabla 1). Analizando el efecto global de la especie en cada fecha de medición sobre el estado fisiológico en términos de fluorescencia de la clorofila se observa que el pino piñonero presenta el menor valor de ФPSII tras el verano (Pvalor $=0.0286$ ), mientras que en las otras tres fechas presentó los valores más altos. En el caso de $\mathrm{Fv} / \mathrm{Fm}$ el comportamiento es similar, identificando en el mes de julio de 2013 valores significativamente superiores para el pino con respecto al resto de especies $(\mathrm{P}$-valor $=0.0232)$.

Tabla 1. Valor promedio de ФPSII y Fv/Fm para las especies (Jt: sabina; Pp: pino piñonero; Qi: encina) y fechas estudiadas (media de todos los tratamientos de luz y exclusión).

\begin{tabular}{|c|c|c|c|c|c|c|c|c|c|c|c|c|}
\hline Fecha & \multicolumn{3}{|c|}{$18 / 07 / 2013$} & \multicolumn{3}{c|}{$23 / 09 / 2013$} & \multicolumn{3}{c|}{$19 / 02 / 2014$} & \multicolumn{3}{|c|}{$28 / 05 / 2015$} \\
espec & $\mathrm{Jt}$ & $\mathrm{Pp}$ & $\mathrm{Qi}$ & $\mathrm{Jt}$ & $\mathrm{Pp}$ & $\mathrm{Qi}$ & $\mathrm{Jt}$ & $\mathrm{Pp}$ & $\mathrm{Qi}$ & $\mathrm{Jt}$ & $\mathrm{Pp}$ & Qi \\
\hline SPSII & 0.685 & 0.703 & 0.672 & 0.604 & 0.523 & 0.589 & 0.535 & 0.576 & 0.543 & 0.699 & 0.706 & 0.681 \\
\hline Fv/Fm & 0.766 & 0.797 & 0.749 & 0.734 & 0.719 & 0.751 & 0.739 & 0.767 & 0.744 & 0.774 & 0.78 & 0.77 \\
\hline
\end{tabular}

Tabla 2. P-valores asociados al ANOVA realizado por especie y fecha para determinar el efecto del nivel de luz y exclusión de precipitación sobre el ФPSII y Fv/Fm

\begin{tabular}{|c|c|c|c|c|c|c|c|}
\hline & & & ФPSII & & & Fv/Fm & \\
\hline & & Jt & $\mathbf{P p}$ & Qi & Jt & $\mathbf{P p}$ & Qi \\
\hline \multirow[t]{3}{*}{ 18/07/2013 } & Luz & $<0.0001$ & $<0.0001$ & 0.0005 & $<0.0001$ & $<0.0001$ & 0.0015 \\
\hline & Exclusión & 0.1640 & 0.2678 & 0.2465 & 0.3070 & 0.0581 & 0.2196 \\
\hline & Luz x Exc & 0.0417 & 0.0760 & 0.2426 & 0.5808 & 0.3164 & 0.3826 \\
\hline \multirow[t]{3}{*}{$23 / 09 / 2013$} & Luz & $<0.0001$ & $<0.0001$ & $<0.0001$ & 0.0003 & 0.1914 & 0.0033 \\
\hline & Exclusión & 0.0983 & 0.4591 & 0.9935 & 0.8686 & 0.2718 & 0.5881 \\
\hline & Luz x Exc & 0.8756 & 0.6928 & 0.2100 & 0.8664 & 0.2364 & 0.01744 \\
\hline \multirow[t]{3}{*}{$19 / 02 / 2014$} & Luz & 0.0002 & 0.0068 & $<0.0001$ & 0.0316 & 0.0079 & 0.0005 \\
\hline & Exclusión & 0.4795 & 0.2168 & 0.3047 & 0.3365 & 0.6563 & 0.0794 \\
\hline & Luz x Exc & 0.0785 & 0.3378 & 0.1637 & 0.0994 & 0.1593 & 0.2524 \\
\hline \multirow[t]{3}{*}{$28 / 05 / 2015$} & Luz & $<0.0001$ & 0.1310 & 0.3567 & 0.0022 & 0.4054 & 0.0831 \\
\hline & Exclusión & 0.0027 & 0.9824 & 0.8790 & 0.0375 & 0.8755 & 0.6250 \\
\hline & Luz x Exc & 0.0135 & 0.2712 & 0.7152 & 0.0162 & 0.9107 & 0.2364 \\
\hline
\end{tabular}

De manera análoga a la supervivencia, se registró un efecto significativo del ambiente lumínico tanto para FPSII como para Fv/Fm (Tabla 2) de tal forma que 
los valores de ambos parámetros disminuyeron al aumentar la luz ( $L L>M L>H L)$. Este efecto se observa en todas las especies y fechas, salvo para el pino piñonero y la encina en la medición de mayo 2015 (ФPSII) y para el piñonero en septiembre 2013 y mayo 2015 (Fv/Fm). Por su parte, no se observa un efecto significativo de la exclusión de la precipitación ni de la interacción luz x exclusión, salvo en el caso de la sabina en las últimas mediciones, indicando, en este caso, un valor más alto de ФPSII en las subparcelas no excluidas, bajo los ambientes lumínicos HL y LL.

Los resultados de la cosecha final mostraron un efecto significativo del ambiente lumínico, de tal manera que en las plantas que sobrevivieron se observó un mayor crecimiento en diámetro, así como mayor biomasa foliar, aérea y total a mayor grado de iluminación (Tabla 3). Por su parte, el efecto de la exclusión sólo se observó en el diámetro, con valores superiores en las plantas control. Por último, se observó un efecto muy significativo de la especie. Así, mientras que el pino piñonero presentó mayores valores asociados a la parte aérea (pesos secos de parte aérea y total, $\mathrm{S} / \mathrm{R}$ y relación de pesos secos de la parte aérea a la total); la encina mostró valores mayores relacionados con el sistema radical (peso seco raíz total y principal, relación de pesos secos de la raíz a la total, SLA); y la sabina albar presentó valores intermedios. Por último, se observó una interacción significativa entre el ambiente lumínico y la exclusión y entre el ambiente lumínico y la especie para varios de los parámetros medidos (Tabla 3, Figura 3).

Tabla 3. Resultados del ANOVA y test de medias de Tukey de los diferentes parámetros morfológicos y alometría estudiados, considerando el efecto del ambiente lumínico (HL, ML y LL), el ensayo de exclusión (excluido (W-) vs no excluido (Co)) y la especie (Jt: sabina; Pp: pino piñonero; Qi: encina) y sus interacciones de primer orden significativas.

\begin{tabular}{|c|c|c|c|c|c|c|c|c|c|c|}
\hline & \multicolumn{3}{|c|}{ Luz } & \multicolumn{2}{|c|}{ Exclusión } & \multicolumn{3}{|c|}{ Especie } & \multirow{2}{*}{$\begin{array}{c}\text { Luz x } \\
\text { exclusión }\end{array}$} & \multirow{2}{*}{$\begin{array}{l}\text { Luz x } \\
\text { especie }\end{array}$} \\
\hline & HL & ML & $\mathbf{L L}$ & & Co & $\mathbf{P p}$ & $\mathbf{J t}$ & Qi & & \\
\hline H & & & & & & $\mathrm{a}$ & $\mathrm{b}$ & $\mathrm{b}$ & & \\
\hline D & $\mathrm{a}$ & b & $\mathrm{c}$ & $\mathrm{b}$ & $\mathrm{a}$ & $\mathrm{a}$ & b & $\mathrm{a}$ & & $* *$ \\
\hline PShojas & $\mathrm{a}$ & $\mathrm{b}$ & $\mathrm{c}$ & & & $\mathrm{c}$ & $\mathrm{a}$ & $\mathrm{b}$ & & \\
\hline PSpaér & $\mathrm{a}$ & b & $\mathrm{c}$ & & & $\mathrm{a}$ & b & b & & $* * *$ \\
\hline PSraíz & & & & & & b & $\mathrm{c}$ & $\mathrm{a}$ & & \\
\hline PStotal & $\mathrm{a}$ & b & c & & & $\mathrm{a}$ & $\mathrm{c}$ & b & & $* *$ \\
\hline $\mathbf{S} / \mathbf{R}$ & $\mathrm{a}$ & $\mathrm{b}$ & $\mathrm{c}$ & & & $\mathrm{a}$ & $\mathrm{b}$ & $\mathrm{c}$ & $*$ & $*$ \\
\hline PShoj/PSpaér & & & & & & $\mathrm{c}$ & $\mathrm{a}$ & $\mathrm{b}$ & & $*$ \\
\hline PSaér/PStot & $\mathrm{a}$ & b & b & & & a & $\mathrm{a}$ & $\mathrm{b}$ & $* *$ & $* * *$ \\
\hline PSrz/PStot & $\mathrm{a}$ & $\mathrm{a}$ & $\mathrm{b}$ & & & b & b & $\mathrm{a}$ & $* *$ & $* * *$ \\
\hline PSrzpp/PSraiz & & & & & & b & c & $\mathrm{a}$ & & \\
\hline PSrz2ª'Praiz & & & & & & b & $\mathrm{a}$ & $\mathrm{c}$ & & \\
\hline SLA & b & b & $\mathrm{a}$ & & & $\mathrm{a}$ & $\mathrm{b}$ & $\mathrm{b}$ & & \\
\hline
\end{tabular}

*** $\mathrm{P}<0.0001 ;{ }^{* *} \mathrm{P}<0.001 ;{ }^{*} \mathrm{P}<0.05$. Diferentes letras muestran diferencias significativas en los niveles de cada efecto. 

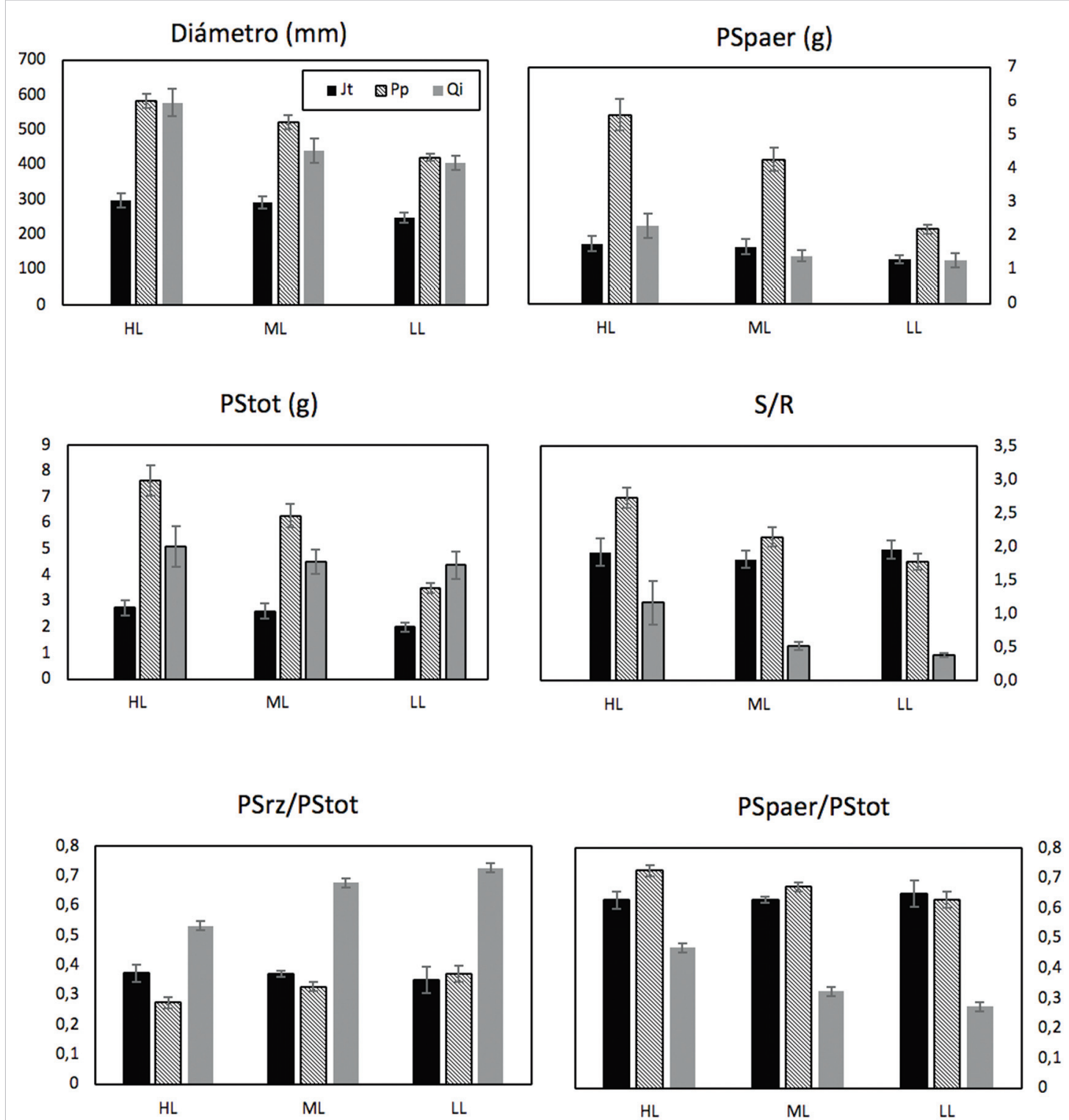

Figura 3. Diagramas de interacción entre el ambiente lumínico (HL: densidad baja; ML, densidad media; LL: densidad alta) y la especie (Jt: sabina; Pp: pino piñonero; Qi: encina).

\section{Discusión}

Este estudio pone de manifiesto el diferente comportamiento morfológico, fisiológico y de supervivencia de las plantas de las tres especies estudiadas en una plantación, bajo ambientes lumínicos diferenciados, considerando el efecto de la exclusión de la precipitación, siendo más notable el efecto de la disponibilidad lumínica que el de la exclusión.

Nuestros resultados corroboran el efecto negativo que el verano (el primero tras la plantación, en mayor medida) tiene sobre la supervivencia de las plantas en 
ambientes mediterráneos continentales (Pardos et al., 2014, Mayoral et al., 2015 y 2016, Calama et al., 2015), ya que la concurrencia de altas temperaturas e irradiaciones elevadas incrementan el efecto negativo de la sequía. Las condiciones estivales limitantes durante el periodo de estudio se vieron agravadas, en mayor medida, durante el verano excepcionalmente seco de 2012, cuando la precipitación estival no superó los $10 \mathrm{~mm}$, valores que se consideran muy escasos para que las plantas lo puedan aprovechar (Vallejo et al., 2012) y que ni aún los dos riegos aplicados lograron paliar.

Aunque la supervivencia de las tres especies estudiadas se vio muy negativamente afectada tanto tras el primer verano, como a lo largo de los 3.5 años de estudio fue, en concreto, la encina la especie que mostró una mayor vulnerabilidad. Resulta especialmente importante que, en los primeros meses tras la plantación, las plantas sean capaces de reanudar su desarrollo radical y alcanzar rápidamente capas del suelo con humedad, siendo éste un mecanismo que refleja la integridad funcional y el vigor de la planta (Grossnickle 2012). La supervivencia de la planta en ambientes mediterráneos está íntimamente relacionada con el crecimiento del sistema radical durante el verano (Villar-Salvador et al., 2012,2013) y antes de que se inicie la sequía estival (Navarro et al., 2006). Nuestros resultados apuntan a que tanto la encina, como el pino piñonero, en menor medida, sufrieron el efecto combinado de las altas temperaturas estivales, la sequía y un sistema radical poco desarrollado fuera del cepellón, lo que contribuyó a las bajas tasas de supervivencia. Al final del ensayo, sin embargo, las plantas de encina que sobrevivieron presentaron un mayor crecimiento del sistema radical con respecto al peso total de la planta que las otras dos especies.

Por su parte, la sabina se caracterizó por un lento crecimiento tanto aéreo como radical, lo que supuso tasas de crecimiento inferiores a las de la encina y el pino piñonero. Esta especie presenta una serie de características que favorecieron unas tasas de supervivencia más elevadas comparadas con la encina y el pino piñonero, tanto durante el primer verano como a lo largo del periodo de estudio. Por una parte, su capacidad para mantener tasas de ganancia de carbono positivas incluso en verano, lo que le supone una ventaja adaptativa (Granda et al., 2014). Por otra, el menor tamaño de planta en el momento de la plantación. En tercer lugar, que su supervivencia no se vea afectada por temperaturas medias diarias superiores a $25^{\circ} \mathrm{C}$ (de Dios García, 2019). Y, por último, su capacidad para colonizar terrenos abandonados por la agricultura, siempre que existan episodios de lluvias esporádicas (Gimeno et al., 2012).

El comportamiento diferenciado de las tres especies en términos de supervivencia se mantuvo bajo los tres niveles de disponibilidad lumínica disponibles, con tasas mayores de supervivencia a mayor densidad de masa (tratamiento LL), mientras que el efecto de la exclusión no resultó muy significativo. De hecho, la exclusión disminuyó la humedad del suelo significativamente durante las épocas de mayor disponibilidad de agua en el suelo (primavera y mediados de otoño), aunque su efecto dejó de ser tan evidente durante el verano y comienzos del otoño, que es cuando la supervivencia resultó más afectada. Durante el verano, el régimen de 
precipitaciones esporádico y escaso en el entorno mediterráneo continental conlleva pérdidas en la intercepción de agua debido a las tasas de evaporación elevadas (Limousin et al., 2008), lo que puede reducir la eficiencia del experimento de exclusión.

El efecto facilitador del arbolado adulto resulta especialmente beneficioso en las etapas iniciales del crecimiento en ambientes mediterráneos (Schupp, 1995; Ledo et al., 2014), ya que el exceso de luz puede resultar un factor de estrés importante (Gómez-Aparicio et al., 2006). En general, las plantas bajo sombra ven reducido el sobrecalentamiento, el déficit de presión de vapor y el estrés oxidativo (Sack et al., 2003), aunque algunas adaptaciones al estrés hídrico pueden oponerse a las condiciones de sombra, si estas son muy intensas (Jiménez et al., 2009). Esas condiciones no se dan en nuestro estudio, donde el ambiente de mayor sombra (tratamiento LL) no es tan intenso $(\mathrm{GSF}=0.25)$. En cualquier caso, el efecto beneficioso del ambiente más sombreado sobre la supervivencia de las plantas de las tres especies estudiadas no radica tanto en una mayor humedad en el suelo, como en unos niveles de radiación y temperaturas de suelo no tan elevados. Dichos niveles disminuyen la demanda evaporativa y reducen el estrés hídrico, a pesar de la baja disponibilidad de agua en el suelo, favoreciendo la supervivencia de las plantas (Gómez-Aparicio et al., 2006).

Estudios anteriores han demostrado las bajas tasas de supervivencia del regenerado del pino piñonero y la encina en espacios abiertos, sobre todo en plantaciones (Pardos et al., 2014; Ogaya y Peñuelas, 2007). En concreto, la regeneración del pino piñonero en la Meseta Norte requiere ambientes de media sombra para optimizar su nicho de regeneración, en términos de supervivencia y asimilación de carbono (Calama et al., 2015). Por su parte, la sabina es una especie que presenta dificultades de emergencia, sin embargo, su probabilidad de establecimiento es alta una vez que se ha producido dicha emergencia (Granda et al., 2014). Es por ello que se requieren unas condiciones medias de luz con GSF inferiores a 0.75 para asegurar la supervivencia de las tres especies estudiadas, siendo la encina más exigente a este respecto (GSF 0.26), que el pino o la sabina (GSF 0.45 a 0.75).

La supervivencia de las plantas de las tres especies estuvo íntimamente relacionada con su comportamiento fisiológico, que a su vez se vio modificado por el ambiente lumínico y por la época del año, pero no por la exclusión. Los valores registrados de $\mathrm{Fv} / \mathrm{Fm}$ por debajo de 0.75 , en verano, otoño e invierno, fueron un signo de fotoinhibición (Ogaya et al., 2011). En concreto, la encina fue la especie que presentó en verano un peor estado fisiológico, en términos de fluorescencia a la clorofila, principalmente en las plantas crecidas a pleno sol (tratamiento HL), lo que se tradujo en la elevada mortalidad. Por su parte, la sabina presentó una mayor plasticidad a los tratamientos, con una ligeramente mayor sensibilidad al frío, lo que le confiere una ventaja adaptativa. Las altas irradiaciones junto con la baja disponibilidad hídrica incrementa el efecto negativo de la sequía sobre las plantas, lo que conduce a la fotoinhibición y a una limitación en la asimilación de carbono (Valladares et al., 2005), lo que lleva al fallo hidraúlico y finalmente conduce a la muerte de las plantas (Calama et al., 2015). Resulta interesante destacar los valores 
bajos de Fv/Fm y ФPSII que se observaron al final de verano en las tres especies cuando crecieron a pleno sol. En estas plantas, el efecto de la sequía estival se agudizó con el inicio de un otoño seco, que no permitió la recuperación fisiológica de las mismas. Este hecho es especialmente notable en la sabina y el pino, que disminuyeron sus valores de $\mathrm{Fv} / \mathrm{Fm}$ con respecto al verano, mientras que la encina los incrementó ligeramente. Esta ausencia de reducción en Fv/Fm en la encina al principio del otoño puede deberse a la pérdida de hojas en verano por efecto de la sequía (Ogaya et al., 2011). Por su parte, las plantas de las tres especies crecidas en la sombra (tratamiento LL) mantuvieron unos valores de Fv/Fm relativamente constantes a lo largo del periodo de estudio.

Al final del ensayo (3.5 años después de la plantación) y contrariamente a lo esperado (p.ej. Gómez-Aparicio et al., 2006; Mayoral et al., 2016, Poorter et al., 2012) se observó una mayor inversión en la parte aérea en las plantas de las tres especies (principalmente en el pino y la encina) expuestas a pleno sol (tratamiento HL). Por su parte, el peso del sistema radical varió con la especie, pero no con la luz. Esto supone que las diferencias encontradas en el peso total, S/R, PSpaer/PStotal y PSrz/PStotal fueron debidas a las diferencias en el peso de la parte aérea. En la cosecha final quedaron también reflejadas las diferencias ya conocidas (Mayoral et al., 2016) en el desarrollo morfológico de las tres especies estudiadas, con una clara diferenciación entre las coníferas y la frondosa. Así, se observó una mayor inversión en la parte aérea en el pino, y mayor inversión en la parte radical en la encina, con valores intermedios en la sabina. Resulta interesante destacar también cómo la luz modificó el efecto de la especie para el diámetro, PSpaér, PStot, S/R, PSpáer/PStot y PSrz/PStot. Así, el diámetro se mantuvo relativamente constante con independencia de la luz en la sabina, mientras que fue disminuyendo al hacerlo la disponibilidad de luz en las otras dos especies. Por su parte, el peso seco de la parte aérea disminuyó al hacerlo la luz en el pino, pero se mantuvo relativamente constante en el pino y la encina. Por último, PSpáer/PStot disminuyó y PSrz/PStot aumentó sólo en la encina al disminuir la cantidad de luz.

Nuestros resultados ponen de manifiesto un peor comportamiento en términos de supervivencia, crecimiento y fisiología de la encina frente a las otras dos especies. Sin embargo, la capacidad rebrotadora de esta especie probablemente le conferirá una ventaja ecológica adaptativa. A través del sistema radical extenso y una parte aérea pequeña rebrotadora podrá usar las reservas del suelo y desplegar una mayor capacidad para asimilar el agua y los nutrientes disponibles (Moreira et al., 2009, Guárdia et al., 2012). Por su parte, la mayor plasticidad de las plantas de la sabina (en términos fisiológicos) y de la encina (en términos de alometría) a diferentes ambientes de luz y disponibilidad hídrica, así como su capacidad para aprovechar las lluvias esporádicas durante el verano, les conferirán una ventaja adaptativa a estas dos especies, una vez que el regenerado está establecido. Por tanto, cabe esperar que la persistencia potencial a medio-largo plazo de las tres especies en la Meseta Norte resultarán especialmente negativa para el pino piñonero. No hay que olvidar, sin embargo, que la previsión de una mayor frecuencia de episodios de sequía y su alargamiento durante parte del otoño en los ecosistemas mediterráne- 
os, reducirá la resiliencia de la encina, al verse posiblemente afectada su capacidad rebrotadora (Barbeta y Peñuelas, 2016). Además, existen evidencias de que la mayor duración de la sequía será el factor que más negativamente afecte la persistencia de estos ecosistemas (Barbeta y Peñuelas, 2016).

\section{Conclusiones}

La supervivencia y comportamiento de la plantación de las tres especies estudiadas están íntimamente relacionadas con la cobertura arbórea, requiriéndose unas condiciones medias de luz con GSF inferiores a 0.75 . Dicha supervivencia está ligada con el comportamiento fisiológico de las especies, que a su vez se vio modificado en función del ambiente lumínico y la época del año, y no tanto por la exclusión.

Las mayores tasas de supervivencia se obtuvieron para las tres especies bajo las cubiertas arbóreas más densas. Este efecto beneficioso del ambiente más sombreado radica en unos niveles de radiación y temperaturas de suelo no tan elevados bajo sombra, más que en una mayor humedad del suelo.

Nuestros resultados muestran un peor comportamiento de la encina frente a las otras dos especies, en términos de supervivencia, crecimiento y fisiología. Sin embargo, la capacidad rebrotadora de la encina, junto con su mayor plasticidad en términos de reparto de biomasa a diferentes ambientes de luz y disponibilidad hídrica le conferirán una ventaja ecológica adaptativa frente al pino piñonero. Esta ventaja adaptativa frente al pino piñonero también la muestra la sabina debido a su mayor plasticidad fisiológica, así como a su capacidad para aprovechar las lluvias esporádicas durante el verano. Es por ello que la persistencia potencial a mediolargo plazo de las tres especies en la Meseta Norte resultarán especialmente negativa para el pino piñonero. No hay que olvidar, sin embargo, la reducción de la resiliencia de la encina al poderse ver afectada su capacidad rebrotadora bajo episodios de sequía más frecuentes y que se alargan durante parte del otoño.

\section{Agradecimientos}

Este trabajo se ha financiado con los proyectos AGL-83828-C2.1R, AGL-201015521, RTA-2013-00011-C02-01 y AT-2013-004. Agradecemos al Servicio Territorial de Medioambiente de Valladolid su ayuda para realizar el ensayo y a Mar Conde y Enrique Garriga su ayuda en el trabajo de campo.

\section{Bibliografía}

Barbeta, A.; Peñuelas, J.; 2016. Sequence of plant responses to droughts of different timescales: lessons from holm oak (Quercus ilex) forests. Plant Ecology \& Diversity. 9 (4), 321-338. https://doi.org/10.1080/17550874.2016.1212288 
Besson, C.K.; Lobo-do-Vale, R.; 2014. Cork oak physiological responses to manipulated water availability in a Mediterranean woodland. Agricultural and Forest Meteorology 184, 230-242. https://doi.org/10.1016/j.agrformet.2013.10.004

Calama, R.; Puértolas, J.; Manso, R.; Pardos, M.; 2015. Defining the optimal regeneration niche for Pinus pinea L. through physiology-based models for seedling survival and carbon assimilation. Trees - Struct. Funct. 29, 1761-1771. https://doi.org/10.1007/ s00468-015-1257-5

de Dios García, J.; 2019. Estudio de las masas mixtas de Pinus pinea L. en la Meseta Norte. Crecimiento y dinámica en un contexto de cambio global. Tesis doctoral, $228 \mathrm{p}$

de Dios García, J.; Manso, R.; Calama, R.; Fortin, M.; Pardos, M.; 2018. A new multifactorial approach for studying intra-annual secondary growth dynamics in Mediterranean mixed-forests: integrating biotic and abiotic interactions. Can J For Res 48:1-12. https://doi.org/10.1139/cjfr-2017-0139

Gómez-Aparacio, L.; Valladares, F.; Zamora, R.; 2006. Differential light responses of Mediterranean tree saplings: linking ecophysiology with regeneration niche in four cooccurring species. Tree Physiology 26: 947-958. https://doi.org/10.1093/treephys/26. 7.947

Gimeno, T.E.; Camarero, J.J.; Granda, E.; Pías, B.; Valladares, F.; 2012. Enhanced growth of Juniperus thurifera under a warmer climate is explained by a positive carbon gain under cold and drought. Tree Physiol. 32, 326-336. https://doi.org/10.1093/tree phys/tps011

Granda, E.; Escudero, A.; Valladares, F.; 2014. More than just drought: complexity of recruitment patterns in Mediterranean forests. Oecologia 176, 997-1007. https://doi. org/10.1007/s00442-014-3064-x

Grossnickle, S.C.; 2012. Why seedlings survive: influence of plant attributes. New Forests 43, 711-738. https://doi.org/10.1007/s11056-012-9336-6

Guàrdia, M.; Fernández, J.; Elena, G.; Fleck, I.; 2012. Stomatal patchiness in the Mediterranean holm oak (Quercus ilex L.) under water stress in the nursery and in the forest. Tree Physiology 32, 829-838. https://doi.org/10.1093/treephys/tps035

Jiménez, M.D.; Pardos, M.; Puértolas, J.; Kleczkowski, L.A.; Pardos, J.A.; 2009. Deep shade alters the acclimation response to moderate water stress in Quercus suber L. Forestry 82(3), 285-298. https://doi.org/10.1093/forestry/cpp008

Kerr. K.L.; Meinzer, F.C.; McCulloh, K.A.; Woodruff, D.R.; Marias, D.E.; (2015. Expression of functional traits during seedling establishment in two populations of Pinus ponderosa from contrasting climates. Tree Physiol 35: 535-548. https://doi.org/10.109 3/treephys/tpv034

Kramer, D.M.; Johnson, G.; Kiirats, O.; Edwards, G.E.; 2004. New fluorescence parameters for the determination of QA redox state and excitation energy fluxes. Photosynthesis Res 79(2): 209-218. https://doi.org/10.1023/B:PRES.0000015391.99477.0d

Ledo, A.; Cañellas, I.; Barbeito, I.; Gordo, F.J.; Calama, R.; Gea-Izquierdo, G.; 2014. Species coexistence in a mixed Mediterranean pine forest: Spatio-temporal variability in trade-offs between facilitation and competition. For. Ecol. Manage. 322, 89-97. https:// doi.org/10.1016/j.foreco.2014.02.038

Leuzinger, S.; Luo, Y.; Beier, C.; Dieleman, W.; Vicca, S.; Körner, C.; 2011. Do global change experiments overestimate impacts on terrestrial ecosystems? Trends Ecol Evol 26(5): 236-241. https://doi.org/10.1016/j.tree.2011.02.011 
Limousin, J.C.; Longepierre, D.; Huc, R.; Rambal, S.; 2010. Change in hydraulic traits of Mediterranean Quercus ilex subjected to long-term throughfall exclusion. Tree Physiol 30, 1026-1036. https://doi.org/10.1093/treephys/tpq062

Limousin, J.C.; Rambal, S.; Ourcival, J.M.; Joffre, R.; 2008. Modelling rainfall interception in a mediterranean Quercus ilex ecosystem: Lesson from a throughfall exclusion experiment. Journal of Hydrology 357(1-2), 57-66. https://doi.org/10.1016/j.jhydrol.2008.05.001

MacKay, S.L.; Arain, M.A.; Khomik, M. et al., 2012. The impact of induced drought on transpiration and growth in a temperate pine plantation forest. Hydrolog. Procc. 26, 17 79-1791. https://doi.org/10.1002/hyp.9315

Manso, R.; Pukkala, R.; Pardos, M.; Miina, J.; Calama, R.; 2014. Modelling Pinus pinea forest management to attain natural regeneration under present and future climatic scenarios. Can J For Res 44:250-262. https://doi.org/10.1139/cjfr-2013-0179

Martin-StPaul, N.K.; Limousin, J.M.; Vogt-Schilb, et al., 2013. The temporal response to drought in a Mediterranean evergreen tree: comparing a regional precipitation gradient and a throughfall exclusion experiment. Global Ch Biol 19, 2413-2426. https://doi.org/ $10.1111 / \mathrm{gcb} .12215$

Mayoral, C.; Calama, R.; Sánchez-González, M.; Pardos. M.; 2015. Modelling the influence of light, water and temperature on photosynthesis in young trees of mixed Mediterranean forests. New Forests 46, 485-506. https://doi.org/10.1007/s11056-015-9471-y

Mayoral, M.; Pardos, M.; Sánchez-González, M.; Brendel, O.; Pita, P.; 2016. Ecological implications of different water use strategies in three coexisting mediterranean tree species. For Ecol Manag 382, 76-87. https://doi.org/10.1016/j.foreco.2016.10.002

Moreira, F.; Catry, F.; Lopes, T.; Bulgalho, M.N.; Rego, F.; 2009. Comparing survival and size of resprouts and planted trees for post-fire forest restoration in central Portugal. Ecological Engineering 35, 870-873. https://doi.org/10.1016/j.ecoleng.2008.12.017

Navarro, R.M.; Del Campo, A.; Cortina, J.; 2006. Factores que afectan al éxito de una repoblación y su relación con la calidad de planta. En: "Calidad de planta forestal para la restauración en ambientes Mediterráneos" (Cortina, J.; Peñuelas, J.L.; Puértolas, J.; Savé, R.; Vilagrosa, A. eds). M Medio Ambiente, Madrid, Spain, pp. 31-46.

Ogaya, R.; Peñuelas, J.; 2007. Tree growth, mortality, and above-ground biomass accumulation in a holm oak forest under a five-year experimental field drought. Plant Ecology 189, 291-299. https://doi.org/10.1007/s11258-006-9184-6

Ogaya, R.; Peñuelas, J.; Asensio, D.; Llusià, J.; 2011. Chlorophyll fluorescence responses to temperature and water availability in two co-dominant Mediterranean shrub and tree species in a long-term field experiment simulating climate change. Environmental and Experimental Botany 71 (2), 123-127. https://doi.org/10.1016/j.envexpbot.2010.10. 016

Pardos, M.; Puértolas, J.; Madrigal, G.; Garriga, E.; de Blas, S.; Calama, R; 2011. Seasonal changes in the physiological activity of regeneration under a natural light gradient in a Pinus pinea regular stand. For Syst 19(3). https://doi.org/10.5424/fs/2010193-9102

Pardos, M.; Calama, R.; 2017. Responses of Pinus pinea seedlings to moderate drought and shade: is the provenance a differential factor? Photosynthetica 55: 1-13. https://doi.org/ 10.1007/s11099-017-0732-1

Pardos. M.; Calama, R.; Mayoral, M.; Madrigal, G.; Sánchez-González, M.; 2014. Addressing post-transplant summer water stress in Pinus pinea and Quercus ilex seedlings. iForest - Biogeosciences and Forestry 8, 348-358. https://doi.org/10.3832/ifor1256-007 
Poorter, H.; Niklas, K.J.; Reich, P.B.; Oleksyn, J.; Poot, P.; Mommer, L.; 2012. Biomass allocation to leaves, stems and roots: meta-analyses of interspecific variation and environmental control. New Phytol 193: 30-50. https://doi.org/10.1111/j.1469-8137. 2011.03952.x

Quero, J.L.; Villar, R.; Marañón, T.; Zamora, R.; 2006. Interactions of drought and shade effects on four Mediterranena Quercus species: physiological and structural leaf responses. New Phytol 170: 819-834. https://doi.org/10.1111/j.1469-8137.2006.01713.x

Rustad, L.E.; 2008. The response of terrestrial ecosystems to global climate change: towards an integrated approach. Sci Total Environ 404: 222-235. https://doi.org/10.1016/j.scito tenv.2008.04.050

Sack, L.; Grubb, P.J. and Marañón, T.; 2003 The functional morphology of juvenile plants tolerant of strong summer drought in shaded forest understories in southern Spain. Plant Ecol. 168, 139 - 163. https://doi.org/10.1023/A:1024423820136

Saunier, A.; Ormeño, E.; Havaux, M. et al., 2018. Resistance of native oak to recurrent drought conditions simulating predicted climatic changes in the Mediterranean region. Plant, Cell Environ. 41, 2299-2312. https://doi.org/10.1111/pce.13331

Schupp, E.W.; 1995. Seed-seedling conflicts, habitat choice, and patterns of plant recruitment. American Journal of Botany 82, 399-409. https://doi.org/10.1002/j.1537-2197. 1995.tb12645.x

Sperlich, D.; Chang, C.T. et al., (2015) Seasonal variability of foliar photosynthetic and morphological traits and drought impacts in a Mediterranean mixed forest. Tree Physiol 35: 501-520. https://doi.org/10.1093/treephys/tpv017

Smith, M.D.; Knapp, A.K.; Collins, S.L.; 2009. A framework for assessing ecosystem dynamics in response to chronic resource alterations induced by climate change. Ecology 90: 3279-3289. https://doi.org/10.1890/08-1815.1

Vallejo, V.R.; Smanis, A.; Chirino, E.; Fuentes, D.; Valdecantos, A.; Vilagrosa, A.; 2012. Perspectives in dryland restoration: approaches for climate change adaptation. New Forests 43, 561-579. https://doi.org/10.1007/s11056-012-9325-9

Villar-Salvador, P.; Puértolas, J.; Cuesta, B.; Peñuelas, J.L.; Uscola, M.; Heredia-Guerrero, N.; Rey Benayas, J.M.; 2012. Increase in size and nitrogen concentration enhances seedling survival in Mediterranean plantations. Insights from an ecophysiological conceptual model of plant survival. New Forests 43 (5-6), 755-770. https://doi.org/10.1007 /s11056-012-9328-6

Villar-Salvador P.; Peñuelas, J.L.; Jacobs, D.F.; 2013. Nitrogen nutrition and drought hardening exert opposite effects on the stress tolerance of Pinus pinea L. seedlings. Tree Physiology 33 (2), 221- 232. https://doi.org/10.1093/treephys/tps133

Valladares, F.I.; Dobarro, I.; Sánchez-Gómez, D.; Pearcy, R.W.; 2005. Photoinhibition and drought in Mediterranean woody samplings: scaling effects and interactions in sun and shade phenotypes. Journal of Experimental Botany 56, 483 - 494. https://doi.org/10. 1093/jxb/eri037 